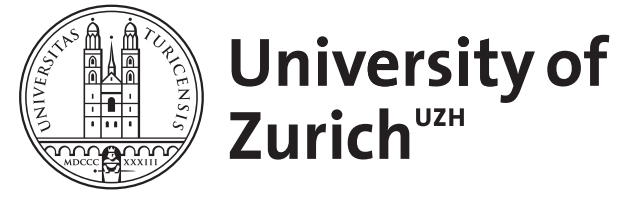

Zurich Open Repository and Archive

University of Zurich

University Library

Strickhofstrasse 39

CH-8057 Zurich

www.zora.uzh.ch

Year: 2017

\title{
Poststrukturalistische Theorie
}

Berndt, Frauke ; Dolar, Mladen

DOI: https://doi.org/10.1515/9783110332681-005

Posted at the Zurich Open Repository and Archive, University of Zurich

ZORA URL: https://doi.org/10.5167/uzh-162022

Book Section

Published Version

Originally published at:

Berndt, Frauke; Dolar, Mladen (2017). Poststrukturalistische Theorie. In: Berndt, Frauke; Goebel, Eckart. Handbuch Literatur Psychoanalyse. Berlin, Boston: De Gruyter, 92-109.

DOI: https://doi.org/10.1515/9783110332681-005 


\title{
II.4. Poststrukturalistische Theorie
}

\author{
Frauke Berndt und Mladen Dolar
}

\section{Einleitung}

Der Begriff ,Poststrukturalismus' ist von einem Paradox geprägt: Niemand hat sich je selbst als Poststrukturalistin oder Poststrukturalisten bezeichnet. Der Begriff wurde zu Beginn der späten 1970er Jahre hauptsächlich in den USA geprägt und hatte in den 1980er Jahren Hochkonjunktur. Da nahezu alle Anhängerinnen und Anhänger dieser Strömung in Frankreich beheimatet waren, fungierte der Begriff auch als Kurzformel für die ,Französische Theorie‘ (vgl. Cusset 2003). Angesichts der dramatischen Entwicklungen der französischen Theoriebildung der 1960er und 1970er Jahre darf man allerdings daran zweifeln, dass Jacques Lacan, Michel Foucault, Jacques Derrida, Gilles Deleuze, Julia Kristeva, Roland Barthes, Jean-François Lyotard, Jean Baudrillard und andere zu ein und derselben Schule oder philosophischen Bewegung gehören, denn sie unterschieden sich in ihren Formen, Hintergründen und Motivationen. Darüber hinaus gab es zwischen ihnen zahlreiche Auseinandersetzungen (vgl. Macksey 1972). Obwohl man mit dem Begriff also erhebliche Vereinfachungen in Kauf zu nehmen hat, markiert das Label ,Poststrukturalismus' jedoch den großen intellektuellen Umbruch - eine Revolution in den Humanwissenschaften, zu der Ferdinand de Saussure das Vorspiel bildet und deren Nachspiel noch heute andauert. Alain Badiou hat dieses ,französische Moment' sogar mit den großen Momenten konzeptueller Dichte in der Philosophiegeschichte verglichen, die sich innerhalb eines Jahrhunderts in der Blütezeit griechischen Denkens und des halben Jahrhunderts im deutschen Idealismus abspielten (vgl. Badiou 2012).

Vor allem ist der Gebrauch des Präfixes ,post' am Begriff eher das Anzeichen eines Dilemmas als ein konzeptueller Schachzug. Man tendiert dazu, das, ,was danach kommt', in Relation zu dem, ,was davor war', zu setzen, wenn man nicht fähig ist, das Neue mit einem eigenen Begriff zu definieren. Das Gleiche gilt übrigens für den Begriff ,Postmoderne', der ein ,Post‘ zur ,Moderne‘ behauptet, diese indes fortsetzt, wiederholt, verdrängt, verleugnet oder überbietet $(\rightarrow$ IV.11. LITERATUR DER MODERNE). Die Sache wird dadurch nicht besser, dass bereits ,Strukturalismus' ein von außen verfügtes Label ist, das ein Großteil der Theoretikerinnen und Theoretiker ablehnte, auf die es in den frühen 1960er Jahren zunächst

Übersetzung: Aus dem Englischen übersetzt von David Pister

https://doi.org/10.1515/9783110332681-005

Bereitgestellt von | UZH Hauptbibliothek / Zentralbibliothek Zürich

Angemeldet

Heruntergeladen am | 14.02.19 11:03 
angewendet wurde. Als der Begriff dieses Mal von Frankreich aus in Umlauf gebracht wurde, versprach er den Geistes- und Sozialwissenschaften auf der Grundlage der strukturellen Linguistik nichts Geringeres als eine neue Epistemologie ( $\rightarrow$ II.1. SEMIOTIK). Insbesondere Claude Lévi-Strauss gilt als bedeutende Figur dieses Umschlags. Dass dieses neue Paradigma die traditionellen Annahmen des Humanismus ablöst, fasst Foucault 1966 unter dem Motto vom ,Tod des Menschen' zusammen. Wichtig sind in diesem Zusammenhang auch Louis Althussers strukturalistische Reformulierung des Marxismus sowie Lacans strukturalistische Wende der Psychoanalyse, die beide im weitesten Sinne als ,antihumanistisch' gelten können. Hinzu treten radikale literarische Praktiken und neue Auffassungen über die Literaturwissenschaft - insbesondere repräsentiert von Tel Quel (Kristeva, Barthes u. a.), sodass die vermeintlichen Poststrukturalisten in den 1960er Jahren bekannte Vertreterinnen und Vertreter des Strukturalismus waren, lange bevor der Begriff Poststrukturalismus überhaupt erfunden war. Sie setzen die Debatte um die Epistemologie der Geisteswissenschaften und die Natur der Semiologie fort - nicht ohne Schlussfolgerungen zu ziehen, die weit über den ursprünglichen Anwendungsbereich des Strukturalismus hinausgingen -, und zwar mit radikal anderen Methoden.

Die Psychoanalyse steht im Zentrum dieser Geschichte, auch wenn sie eher eine Frontlinie als einen gemeinsamen Nenner markiert. Dass ausgerechnet Lacan als einer der Hauptvertreter des Poststrukturalismus gilt, ist besonders paradox. Denn er begann seine Karriere sowohl als praktizierender Analytiker als auch als Theoretiker der Psychoanalyse bereits in den 1930er Jahren und produzierte den Großteil seines Werkes in den 1950er Jahren, bevor der ,Strukturalismus' überhaupt in der Öffentlichkeit zirkulierte, vom ,Poststrukturalismus“ ganz zu schweigen. Keiner der beiden Begriffe wird daher auch der bemerkenswerten Kontinuität seines Wirkens seit den späten 1940er Jahren gerecht. Aus der ganzen Reihe von Poststrukturalistinnen und Poststrukturalisten ist er jedoch der Einzige, der eine ungetrübte Loyalität zu Freud und dessen Vermächtnis bezeugt, indem er diese Treue durch radikale Innovation ausdrückt. Im Folgenden soll gezeigt werden, dass das Konzept des Phallus den gemeinsamen Nenner von Literatur, Psychoanalyse und Poststrukturalismus bildet, indem zunächst die poststrukturalistische Kritik an der ödipalen Matrix der Psychoanalyse skizziert wird (vgl. Abschnitt 2.). Vor diesem Hintergrund werden Deleuze' Kafka- und Barthes' Balzac-Lektüren (vgl. Abschnitt 3.) sowie Lacans Poe-Lektüre vorgestellt (vgl. Abschnitt 4.), die eine große Kontroverse auslöste (vgl. Abschnitt 5.). Am Schluss steht eine Bilanz im Hinblick auf die poststrukturalistische ,Phallogozentrismuskritik' (vgl. Abschnitt 6.). 


\section{Kritik: Foucault - Deleuze - Derrida}

Das Zentrum der poststrukturalistischen Auseinandersetzung mit der Psychoanalyse bildet die Kritik an deren ödipaler Matrix ( $\rightarrow$ III.6. ÖDIPUS). Foucault tritt 1961 in Histoire de la folie (Wahnsinn und Gesellschaft) mit einer harschen Kritik der Freud'schen Psychoanalyse an, die er als Fortsetzung, als neue und gedämpfte Form des Asyl- und Medizinsystems interpretiert. In Les mots et les choses (1966; Die Ordnung der Dinge) wird man indes von seiner Begeisterung für die Psychoanalyse überrascht, die - trotz einer gewissen Distanz - nun als Teil der Lösung eben dieses Systems gilt. Gegen Ende seines Schaffens können die drei Bände der Histoire de la sexualité (1976-1984; Sexualität und Wahrheit) dann wiederum als langer Kreuzzug gegen die Psychoanalyse, insbesondere gegen die Art und Weise, wie sie der Gesellschaft ihre Ansichten über Sexualität als allgemeingültiges Bezugssystem aufdrängt, verstanden werden. Die Psychoanalyse erscheint als Foucaults bevorzugter Gegner, gewissermaßen als sein Lieblingsfeind, sodass sein gesamtes Werk retrospektiv als kontinuierlicher Versuch gelesen werden kann, ein alternatives konzeptuelles und praktisches Bezugssystem zur Verfügung zu stellen. Nebenbei bemerkt: Foucault, der sorgfältige Prüfer der Archive, zitiert in all seinen kritischen Auseinandersetzungen weder Freud noch Lacan. Lediglich in seinem ersten publizierten Text bezieht er sich in der Einführung zu seiner französischen Übersetzung von Ludwig Binswangers Traum und Existenz aus dem Jahr 1954 unmittelbar auf Freud. Foucaults Feind ist also von Anfang an immer das Bild der Psychoanalyse im Zeitgeist, ihre Sprache, ihr weitverbreiteter Einfluss, ohne dass Foucault jemals ihre epistemologischen Grundsätze beachtet oder ihre Konzepte analysiert hat. Obwohl also Freud für ihn als Strohmann fungiert, verliert Foucaults Kritik an der Art und Weise, in der Psychoanalyse in der Gesellschaft allgemeine Wahrnehmungen formt, freilich nicht ihre Gültigkeit.

Deleuze' Auseinandersetzung mit Freud zeichnet eine noch stärkere Ambivalenz aus: In Différence et répétition (1968; Differenz und Wiederholung) wird er als der heroische, konzeptuelle Erfinder der Wiederholung in einer posthegelianischen Philosophie bejubelt, wobei Søren Kierkegaard und Friedrich Nietzsche ihm bei dieser Denkfigur den Weg bereitet haben. Das Konzept des Todestriebes bildet ( $\rightarrow$ IV.1. TRAGöDIE) - zusammen mit dem Konzept der ewigen Wiederkehr den Kern von Deleuze' Argumentation. Im letzten Teil der Logique du sens (1969; Logik des Sinns) stellt er eine breite Exegese psychoanalytischer Konzepte vor darunter am prominentesten das kontrovers diskutierte Konzept des Phallus. Obwohl Deleuze das Gegenteil behauptet, handelt es sich kurioserweise um den ,phallozentrischsten' Entwurf aus poststrukturalistischem Umfeld. Nur wenige Jahre trennen diese beiden Bücher vom zusammen mit Félix Guattari verfassten L’Anti-Edipe (1972; Anti-Ödipus), einer der wohl spektakulärsten Attacken auf das 
psychoanalytische Unterfangen als Ganzes, obwohl der terminologische Apparat des Buches - insbesondere die Begehrensmaschine - einen Freud'schen Gedankengang mit anderen Mitteln fortsetzt. Lacan bleibt zwar von der Kritik meistens verschont, dennoch beginnt mit dem Buch eine von Foucault in seiner Histoire de la sexualité fortgesetzte Kontroverse, welche die Anhängerinnen und Anhänger Foucaults und Deleuze' gegen diejenigen Lacans aufbringt (vgl. Schuster 2016).

Im Gegensatz zu Foucault oder Deleuze schwankt Derrida in seiner Bewertung Freuds nicht zwischen zwei Extremen, sondern historisiert die Psychoanalyse von Beginn an. Einerseits achtet er sie als Wegbereiter für die Dekonstruktion, die, Philosophie und Kunst zusammenführend, die Grenzen des metaphysischen Paradigmas vermisst. In seinem Essay Freud et la scène de l'écriture (Freud und der Schauplatz der Schrift) weist Derrida bereits 1967 mit Nachdruck darauf hin, dass Freud in seinem Text über den Wunderblock (1925) das Modell eines dezentrierten Subjekts entwirft, das hauptsächlich von der Schrift und der Spur abhängt, diesen Überlegungen allerdings nicht weit genug folgt (vgl. Derrida 1972 [1967]). Andererseits kritisiert er, dass die Psychoanalyse der ödipalen Familienstruktur ebenso verpflichtet bleibt, wie sie die zentralen Positionen des Phallus - scheinbar zu einem bloßen Signifikanten degradiert - und des Mangels bestätigt, der immer noch die Modalität eines - wenn auch leeren - Zentrums bildet. Deshalb argumentiert Derrida 1967 in De la Grammatologie (Grammatologie), dass ,Phallozentrismus' und Logozentrismus im ,Phallogozentrismus' zusammenlaufen ein Begriff, der den Logos und die ödipale Begehrensökonomie gewissermaßen zu Komplizen macht. An diesem Punkt greifen vor allem poststrukturalistische Feministinnen wie Kristeva, Luce Irigaray und Hélène Cixous die Psychoanalyse ebenfalls scharf an ( $\rightarrow$ II.7. GENDER UND QUEER STUDIES).

\section{Subversion: Deleuze - Guattari - Barthes}

Zwar setzen sich nicht wenige Poststrukturalistinnen und Poststrukturalisten mit literarischen Texten auseinander (vgl. Geisenhanslüke 2015) - insbesondere in den 1960er und 1970er Jahren in der Gruppe Tel Qel, so benannt nach der von Philippe Sollers und anderen gegründeten Zeitschrift: Foucault (vgl. During 2012) ebenso wie Lyotard (vgl. Sawyer 2014), mit psychoanalytischem Interesse aber vor allem Deleuze in seiner Lektüre von Leopold Sacher-Masochs Novelle Venus im Pelz (1870) ( $\rightarrow$ IV.6. FALlGESCHICHTE). In seinem Essay De Sacher-Masoch au masochisme (1961) argumentiert er, dass Sadismus und Masochismus keiner einheitlichen Triebökonomie folgten, sondern dass im Masochismus der Phallus annulliert werde, weil die „symbolische Ordnung“ des Masochismus eine „inter- 
maternelle“ (Deleuze 1968 [1961], 222) Ordnung sei. 1975 wendet Deleuze sich dann zusammen mit Guattari in der Studie Kafka, pour une littérature mineure (Kafka: Für eine kleine Literatur) gegen Franz Kafkas ,Ödipalisierung، (vgl. Lecercle 2012), die diesen Autor auf den Familienroman verpflichtet ( $\rightarrow$ IV.5. FAMILIEnRoman). Travestie und Parodie zeichnen die Zerrbilder der Familie, insbesondere aber des aufgeblasenen Vaters und geschrumpften Sohnes sowohl in Kafkas Romanen als auch in seinem fiktionalen Brief an den Vater (1919) aus. Allerdings bemerken sie bald: „Allein der Ausdruck erschließt das Verfahren“ (Deleuze und Guattari 2014 [1975], 24), das - hier folgen sie einem Begriff aus Kafkas Tagebüchern - „kleine[ ] Literaturen“ (Kafka 1983, 154) erzeugt, die Deleuze und Guattari als antiödipale Ausdrucksmaschinen bezeichnen. In ihrer sprachlichen Konfiguration ist diese Literatur in einer anderen Weise als die thematisch orientierte littérature engagée revolutionär:

\footnotetext{
Das also sind die drei charakteristischen Merkmale einer kleinen Literatur: Deterritorialisierung der Sprache, Koppelung des Individuellen ans unmittelbar Politische, kollektive Aussageverkettung. So gefaßt, qualifiziert das Adjektiv ,klein' nicht mehr bloß bestimmte Sonderliteraturen, sondern die revolutionären Bedingungen jeder Literatur, die sich innerhalb einer sogenannten ,großen' (oder etablierten) Literatur befindet. (Deleuze und Guattari 2014 [1975], 27)
}

In seinen 1993 unter dem Titel Critique et clinique (Kritik und Klinik) veröffentlichten Essays wendet sich Deleuze dann außer Kafka noch weiteren ,großen Schriftstellern der Klassischen Moderne - Herman Melville, Walt Whitman, Nietzsche, Martin Heidegger oder Samuel Beckett zu, deren Größe sich in einer Minorisierung beziehungsweise Verkleinerung der Sprache niederschlägt. „Die guten Bücher sind in einer Art Fremdsprache geschrieben“ (zit. Deleuze 2000 [1993], 7) - mit dem Marcel Prousts Contre Sainte-Beuve (postum 1954; Gegen Sainte-Beuve) entliehenen Motto, dass die guten Bücher in einer Art Fremdsprache geschrieben seien, beschreibt er Techniken der Subversion, die lexikalische, grammatische und syntaktische Codes durchbrechen, Techniken des ,Nicht-sprachlich-Werdens', die Sprache ins Stottern bringen. Im Stottern und Stammeln trägt - um mit Lacan zu argumentieren - das Reale den Sieg über das Imaginäre sowie das Symbolische davon ( $\rightarrow$ II.8. MEDIENTHEORIE). Mit Jakobson gesprochen, treten dabei alle anderen Funktionen der Sprache hinter ihrer poetischen Funktion zurück (vgl. Jakobson 1979 [1960]). Im Stotter-Essay Bégaya-t-il (1993; Stotterte er ...) führt Deleuze den ästhetisch-affektiven und damit zugleich politisch-utopischen Gehalt einer solchen ,Fremdsprache' darauf zurück, dass die ,großen` Schriftsteller die Sprache „minorisieren“ (Deleuze 2000 [1993], 148), sodass sie sich in selbstreferenzieller Autonomie entfalten kann: 
Das heißt, dass sich ein großer Schriftsteller stets wie ein Fremder in der Sprache befindet, in der er sich ausdrückt, selbst wenn es seine Muttersprache ist. [...] Er ist ein Fremder in der eigenen Sprache: Er mischt nicht eine andere Sprache seiner eigenen bei, er schneidet vielmehr aus seiner Sprache eine fremde Sprache heraus, die nicht vorher gegeben ist. Die Sprache in ihr selbst schreien, stottern, stammeln, murmeln lassen. (Deleuze 2000 [1993], 148)

Gegen den Phallus richtet auch Barthes seine Lektüren (vgl. Neumann 2014, 27-161). Dabei bleibt die Beziehung des herausragenden Literaturwissenschaftlers und -theoretikers zur Psychoanalyse, obwohl sie einen Schlüssel zu seinem Werk bildet, unentschieden. Er nutzt sie als kritisches, nicht zuletzt auch auf die Psychoanalyse selbstkritisch angewendetes Werkzeug, ohne sich indes ihren Grundsätzen zu verschreiben (vgl. Lindorfer 1998, 16-23) - darin ist er den marxistischen Theoretikerinnen und Theoretikern ähnlich ( $\rightarrow$ II.3. KRITISCHE THEORIE). Barthes dienen psychoanalytische Denkfiguren als Medium der Interpretation. In den Mythologies (1957; Mythen des Alltags) analysiert er beispielsweise das kulturelle Imaginäre der Gesellschaft ( $\rightarrow$ II.5. KULTURTHEORIE). In seiner großen Studie S/Z (1970) interpretiert er Honoré de Balzacs gerahmte Novelle Sarrasine (1830; Sarrasine) aus den Scènes de la vie parisienne (Szenen aus dem Pariser Leben), dem zweiten Band der Comédie humaine, als talking back gegen Jean Rebouls klassisch-freudianische, von Georges Batailles Le Bleu du ciel (1957; Das Blau des Himmels) motivierte Interpretation Sarrasine ou la castration personnifiée (1967) strukturalistisch. Barthes durchque(e)rt den Text, indem er aus fünf Signifikaten beziehungsweise Codes ein semiotisches Raster des Textes bildet: aus dem hermeneutischen (Wahrheit), dem semischen (Denotation), dem proaïretischen („Proaïresis = Fähigkeit, das Ergebnis eines Handelns zu befragen“ (Zanetti 2015, 366)), dem symbolischen (Konnotation) und dem referenziellen Code (Wissen). Balzac erzählt - grob gesprochen - von dem Bildhauer Sarrasine, der sich im Italien des 18. Jahrhunderts in die Sängerin Zambinella verliebt: sowohl in ihre Stimme als auch in ihre körperliche Erscheinung, ohne zu erkennen, dass Zambinella ein Kastrat ist; bevor er sie/ihn mit seinem Degen töten kann, wird Sarrasine selbst ermordet.

Barthes strukturalistische Aktivität (l'activité structuraliste) verdichtet das Anagramm S/Z, das er im 47. Abschnitt analysiert: Der effeminierte Titelheld heißt „SarraSine“, nicht „SarraZine“, wie im Französischen zu erwarten wäre. Für Barthes stellt das $Z$ daher das Zeichen der sexuellen Abweichung dar, die gegen die heteronormative Zensur - symbolisiert durch den Querstrich (/) - verstößt. In der graphischen Spiegelung des $S$ im Z betrachtet Sarrasine ,in Zambinella seine eigene Kastration“: 
[V]om Balzacschen Standpunkt ist dieses Z (das im Namen Balzac ist) der Buchstabe der Abweichung (man vergleiche die Novelle Z. Marcas [1840]); an dieser Stelle schließlich ist das Z der Inauguralbuchstabe der Zambinella, das Initial der Kastration, so daß Sarrasine durch diesen Orthographiefehler, der inmitten seines Namens, seines Körpers auftritt, das zambinellische $\mathrm{Z}$ in seiner wirklichen Natur empfängt, der Verletzung des Mangels. (Barthes 2012 [1970], 110)

Allerdings gilt ausgerechnet diesem Mangel und eben gerade nicht dem Phallus Sarrasines Begehren. Als ein solches Zeichen ist das Z daher gleichzeitig (sinn-)leer wie ästhetisch übervoll. In seiner Replik auf Balzac und Barthes L'hermaphrodite. Sarrasine sculpteur (1987) dreht Michel Serres den Spieß daher um und behauptet: „Sarrasine widersetzt sich der Zambinella wie das S dem Z. Aber der Name der Frau oder des Mannes oder des Kastraten bezeichnet, dem $\mathrm{Z}$ beraubt, beide“ - aus diesem Wortspiel ergibt sich ,ambinella“ als ,beide(s)in-ihr‘. „Sie verkörpert nicht den Mangel, sondern die Fülle. Sie verkörpert den Mangel und die Fülle.“(Serres 1987, 74; Übers. F. B.)

Vom Imaginären vollzieht auch Barthes 1973 die Wende zum Realen, indem er sich von den Inhalten ab- und den Verfahren zuwendet. In Le plaisir du texte (1973; Die Lust am Text), dem sicherlich einflussreichsten literaturtheoretischen Text des Poststrukturalismus, setzt er den Text in einem ersten Schritt mit einem „Gewebe“ gleich, weil diese Metapher die „generative Vorstellung“ zum Ausdruck bringt, „daß der Text durch ein ständiges Flechten entsteht und sich selbst bearbeitet“" (Barthes 1974 [1973], 94). In einem zweiten Schritt vergleicht er ihn mit einem erotischen menschlichen (männlichen) Körper, auf den sich das Begehren richtet: „(Plaisir/Jouissance, Lust/Wollust [...])“ (Barthes 1974 [1973], 8). Mit Lacan gesprochen wird der Text aufgrund dieses doppelten Vergleichs in seiner materialen Medialität zum ,Objekt klein a (objet petit $a$ ), auf das sich das nichtphallische, antiödipale Begehren richtet. Er zeichnet sich nicht durch etwas Substantielles aus, was ,Ich` begehren könnte, sondern durch eine „essentielle Leere, die nach signifikanter Füllung drängt, zum dynamischen Element, das die Fluktuationen innerhalb des Symbolischen unablässig vorantreibt und die produktive wie rezeptive Lust am Text hervorbringt“ (Brune 2003, 177-178). Weil seine Lust etwas Allgemeines meint (,Lustprinzip'), unterscheidet sich die Lust am Text sowohl von etwas Besonderem wie der Pornographie als auch von etwas „Geringfügige[m]“ wie der ,Lust an etwas':

Aber mit dieser Zweideutigkeit muß ich mich abfinden; denn auf der einen Seite brauche ich eine allgemeine ,Lust', um mich auf einen Exzeß des Textes beziehen zu können, auf das, was in ihm jede (soziale) Funktion und jedes (strukturale) Funktionieren sprengt; auf der anderen Seite brauche ich eine besondere ,Lust', den bloßen Teil der allumfassenden Lust, wenn ich die Euphorie, das Erfülltsein, das Behagen (ein Gefühl des Sattseins, in das 
die Kultur leicht eindringt) unterscheiden muß vom Schock, von der Erschütterung, vom Vergehen, die der Wollust eigen sind. (Barthes 1974 [1973], 30)

\section{Poe paradigmatisch: Lacan}

Literatur bildet schließlich einen der wichtigsten Schauplätze der Lacan'schen Psychoanalyse (vgl. Zupančič 2000). In seinem Werk wimmelt es von literarischen Referenzen. Neben zahlreichen Anspielungen sind es vor allem die aufschlussreichen, komplexen und facettenreichen Lektüren klassischer Texte, die im Zentrum seiner Theorie stehen; am berühmtesten: Antigone in seinem Seminar L'éthique de la psychoanalyse (1986; Die Ethik der Psychoanalyse; $\rightarrow$ III.7. ANTIGONE), Hamlet in seinem Seminar Le désir et son interprétation (1958/59; Das Begehren und seine Deutung; $\rightarrow$ III.9. HAMLET) und Claudel Coûfontaines Trilogie in seinem Seminar Le transfert (1991; Die Übertragung). Darüber hinaus gibt es eine kürzere Auseinandersetzung mit Jean Genets Balkon (1957) in seinem Seminar Les formations de l'inconscient (1998; Die Bildungen des Unbewussten), einen André Gide gewidmeten Text in seinen Écrits (1966; Schriften), einen weiteren Marguerite Duras gewidmeten Text (vgl. Lacan 2001) sowie Lacans große Verehrung für James Joyce, die seine gesamte spätere Karriere beeinflusst (vgl. Lacan 2005). Wenn es aber einen Fall gibt, in dem sich Literatur, Psychoanalyse und Poststrukturalismus überschneiden, dann ohne Frage in Lacans Interpretation von Poes 1844 im literarischen Almanach The Gift for 1845 veröffentlichter Detektivgeschichte The Purloined Letter (Der entwendete Brief) ( $\rightarrow$ IV.10. KRIMINALLITERATUR). Lacan widmet dieser Geschichte eine Sitzung in seinem Seminar Le moi dans la théorie de Freud et dans la technique de la psychanalyse (1954/1955; Das Ich in der Theorie Freuds und in der Technik der Psychoanalyse), hält 1956 zwei Vorträge, die er 1957 zur Publikation erweitert; 1966 fügt er in seinen Écrits einen Nachtrag hinzu (vgl. Lacan 1973-1980 [1966]). Dabei misst er seiner Poe-Lektüre einen solch exemplarischen Wert zu, dass er sie an den Anfang der großen Ecrits-Ausgabe stellt und ihr damit den Status eines Modells, vielleicht sogar eines Königswegs zu seiner Auffassung von Psychoanalyse verleiht.

Dabei unterscheidet sich Lacans Herangehensweise stark von den üblichen Wegen und Pfaden, die psychoanalytisch inspirierte Analysen oft einschlagen. Erstens umgeht er den biographischen Ansatz, der - im Fall Poes - von Marie Bonapartes The Life and Works of Edgar Allan Poe: A Psychoanalytic Interpretation (1949; Edgar Poe. Eine psychoanalytische Studie) repräsentiert wird und mit der Suche nach Poes ,mentaler Störung، einen Großteil der frühen Forschung beeinflusst. Zweitens - und das ist wichtiger und bemerkenswerter - besteht Lacan wie Barthes darauf, dass die Analyse vom Signifikanten und nicht von dessen Bedeu- 
tung ausgehen soll. Der Gang, den Poes entwendeter Brief nimmt, ist in diesem Sinne exemplarisch für Lacan: Es ist ein Brief, dessen Inhalt nicht bekannt und schlicht gleichgültig ist. Durch Zirkulieren, durch ständiges Deplatzieren bestimmt er jedoch die subjektive Position der beteiligten Figuren. Er erzeugt Effekte, die unabhängig von seinem Inhalt sind. Daher muss der Verlaufsweg des Signifikanten selbst freigelegt werden, damit er unabhängig von seinem Inhalt als Triebkraft der Erzählung erkennbar wird. Darüber hinaus ist der entwendete Brief gerade dadurch versteckt, dass er für alle offen sichtbar ist und nicht etwa in irgendwelchen Tiefen verborgen wird. Lacan folgt gewissermaßen Poes eigenem Ratschlag: „[T]here is such a thing as being too profound. Truth is not always in a well. In fact, as regards the more important knowledge, I do believe that she is invariably superficial.“ (Poe 1967, 204) Die Erzählung zu interpretieren, heißt deshalb gerade nicht, Bedeutung zu finden und offenzulegen - das übliche Geschäft einer Interpretation -, sondern die strukturellen Determinanten und Effekte des Signifikanten zu entwirren.

Drittens hängt Poes Erzählung von der Wiederholung derselben Situation $a b$, in die jeweils unterschiedliche Gruppen von Figuren involviert sind, deren Positionen wiederum strukturell von derjenigen des Briefes abhängen. In Lacans Lesart hängt die Handlung gerade von dieser Wiederholung ab - nicht von der Wiederholung ein und desselben Ereignisses, sondern von einer Wiederholung, in der sich eine Verschiebung abspielt. Aufgrund dieser Struktur deutet Lacan Poes Erzählung als Allegorie der Psychoanalyse. Denn sie ermöglicht nicht nur eine Analyse im Allgemeinen, sondern deutet auch auf die strukturelle Position des Analytikers im Besonderen hin, die in der Erzählung von C. Auguste Dupin besetzt wird - Detektiv und Analytiker in einer Person. Die zweite Szene, in der sich Dupins Intervention abspielt, deutet Lacan daher als Intervention des Analytikers. Das Wichtigste in der Analyse besteht nicht darin, immer wieder das Gleiche - etwa die gleiche, sich wiederholende Kernphantasie - zu finden, sondern sie schöpft ihre Erkenntnis aus der symbolischen Verschiebung des Signifikanten. Sie entwickelt sich durch strukturelle Verschiebungen, nicht durch das Aufzeigen der gleichen Bedeutung in unterschiedlicher Verkleidung. Die Wiederholung ist also nicht Wiederholung eines Gleichen, Wiederholung einer Identität, sondern gerade das, was in der Wiederholung Differenz erzeugt $(\rightarrow$ II.2. RHETORIK UND POETIK).

Viertens geht es in dieser Erzählung dennoch nicht um so etwas wie ,angewandte Psychoanalyse'. Denn der literarische Text ist ebenso ein Werkzeug, um die Psychoanalyse als psychoanalytische Theorie zu interpretieren, wie die psychoanalytische Theorie ein Werkzeug ist, um den literarischen Text zu interpretieren. Es geht hier also tatsächlich um ,Implikation' statt um ,Applikation': „The methodological stake is no longer that of the application of psychoanalysis to 
literature, but rather, of their interimplication in each other.“ (Felman 1988, 153) Lacan selbst weist immer wieder auf den wichtigsten Punkt seiner Poe-Lektüre hin:

Wenn das, was Freud freigelegt hat [...], einen Sinn hat, dann, weil die Verschiebung des Signifikanten die Subjekte in ihren Handlungen, in ihrem Geschick, in ihren Weigerungen, in ihren Verblendungen, in ihrem Erfolg und ihrem Schicksal ungeachtet ihrer angeborenen Anlagen und ihrer sozialen Erwerbungen, ohne Rücksicht auf den Charakter und das Geschlecht bestimmt, und weil wohl oder übel dem Zug des Signifikanten als Sack und Pack alles psychologisch Gegebene folgt. (Lacan 1973 [1966], 29)

Wenn Lacan diesen Gedanken weiter ausführt, dann greift er dafür auf die literarische Technik der Prosopopoiia (fictio personae) zurück, indem er den personifizierten Signifikanten ,jenseits aller Bedeutungen“ antworten lässt:

,Du glaubst zu handeln, während ich dich bewege an Fäden, mit welchen ich deine Begierden verknüpfe. So nehmen diese zu an Kraft und vermehren sich in die Objekte, die dich an die Zerissenheit [sic] deiner Kinderzeit zurückverweisen. Dies soll dein Festmahl sein bis zur Wiederkehr des Steinernen Gasts, der ich sein werde für dich, wenn du mich rufst. (Lacan 1973 [1966], 40)

Dergestalt ist es der Verlauf des Signifikanten, den niemand wirklich beherrschen kann - nicht einmal der Detektivanalytiker, dessen Position ja von ihm abhängt. Am Ende bestimmt dieser Verlauf unser Unbewusstes, und das heißt unser Schicksal - daher auch Lacans spitze Bemerkung, die im letzten Satz versichert, „ein Brief (eine Letter) erreiche immer seinen (ihren) Bestimmungsort“ (Lacan 1973 [1966], 41).

\section{Phallus: Die Derrida-Lacan-Kontroverse}

Auch Derrida versteht die Poe-Lektüre als Königsweg zur Lacan'schen Psychoanalyse und antwortet auf sie mit einer kritischen Lektüre derselben. Diese Antwort lässt allerdings bis 1975 auf sich warten, bis sich Derrida im Essay Le Facteur de la vérité (Der Facteur der Wahrheit) der Herausforderung stellt und zu zeigen versucht, warum Lacans psychoanalytischer Ansatz problematisch und unzureichend ist und wie sein eigener Ansatz der Dekonstruktion dem Purloined Letter gerecht werden kann. Dieser Moment sollte zum ikonischen Moment des Poststrukturalismus werden: Zwei, große Männer' tragen ihre Kontroverse auf dem Schlachtfeld der Literatur aus. Derridas scharfe Kritik kann stark vereinfacht in vier Punkten zusammengefasst werden: 
Erstens stellt er die Frage nach dem Kontext. Lacan nimmt - so merkt Derrida an - Poes Geschichte nämlich zu ernst, ohne jedoch ihre Stellung innerhalb von Poes Trilogie, insbesondere ihre narrative Rahmung zu berücksichtigen: „Lacan schließt, ohne je ein Wort darüber zu sagen, die textuelle Fiktion aus, in deren Innerem er die sogenannte allgemeine Narration abhebt.“ Dabei gilt es indes zu beachten: „Es gibt ein Geviert, unsichtbar aber strukturell irreduzibel, um die Narration herum. Wo beginnt es? bei der ersten Letter.“ (Derrida 1987 [1975], 206) Denn mit der Rahmung wird die Markierung von Grenzverläufen unmöglich: „Die Gevierte sind stets eingeviert: also durch irgendein Stück ihres Inhalts. Stücke ohne Ganzes, ,Partituren' ohne Ensemble, das ist es, was hier den Traum eines Briefes ohne Teilung, allergisch gegen die Teilung, verspielt.“ (Derrida 1987 [1975], 269) Diese Struktur führt Derrida schließlich zu der Schreibszene: „Verfehlt man die Position des Narrators, seine Einbindung in den Inhalt dessen, was er zu erzählen scheint, so läßt man all das aus, was von der Schriftszene die beiden Dreiecke überbordet.“ (Derrida 1987 [1975], 266)

Derridas erster Einwand besteht also darin, dass eine bloß auf dem Signifikanten beruhende Analyse der Geschichte ein bestimmtes Narrativ und damit eine bestimmte Gesamtheit annimmt, ohne mit der narrativen Rahmung die Literarizität des Textes zu reflektieren, die für Derrida dessen Bedeutung bestimmt. Demgegenüber beachtet die Dekonstruktion einerseits eben genau diese Rahmung, stellt Rahmen und Grenzen infrage, ohne sie jedoch vollends abzuschaffen. Andererseits zielt eine dekonstruktive Lesart - anders als Lacans auf Totalität hinauslaufende Lektüre - auf Partialisierung, Dezentrierung und Fragmentierung der Erzählung ab. Während also der Signifikant zur Totalität führt, führt das Schreiben zur Unmöglichkeit von Totalität.

Zweitens kritisiert Derrida Lacans Beharren darauf, dass der Signifikant zwar frei von Bedeutung sei, aber dennoch Effekte produziere, die einer doppelten Wendung entsprechen. Die Dekonstruktion besteht also auf Resten und Überbleibseln, die nicht in eine einzige Bedeutung integriert werden können, auf der Dissemination von Bedeutung, auf deren Abwesenheit sowie auf andauernde Teilungen ohne Ganzes:

Bisher lassen unsere Fragen vermuten, daß, wenn es etwas gibt wie einen entwendeten Brief, die Falle dessen vielleicht supplementär ist: er hätte keinen festen Platz, nicht einmal den eines abgrenzbaren Lochs oder eines zuweisbaren Fehls. Er fände sich nicht, er könnte immer nicht sich finden, er fände sich in jedem Fall weniger in der versiegelten Schrift, wovon der Narrator die durch das Seminar entzifferte ,Geschichte‘ erzählt, weniger in dem Inhalt der Geschichte als ,in' dem Text, der sich, auf einer vierten Seite, sowohl den Augen Dupins entzieht als auch denen des Psychoanalytikers. Der Rest, das Liegengelassene, das wäre Der entwendete Brief, der Text, der diesen Titel trägt, und dessen Statt, wie die einmal mehr unsichtbaren großen Lettern, nicht ist, wo man sich gefaßt machte, sie zu finden, 
in dem gevierten Inhalt des ,realen Dramas' oder in dem verborgenen und versiegelten Inneren der Novelle von Poe, sondern in diesem und als dieser offene, sehr offene Brief, der die Fiktion ist. (Derrida 1987 [1975], 219)

Drittens evoziert der Brief in Lacans Lesart für Derrida den Phallus als zentralen psychoanalytischen Grundsatz. Gerade durch seine mangelnde Bedeutung wird er zum ,transzendentalen Signifikanten', der Bedeutung auf alles andere übertragen kann. Er evoziert die Kastration und stellt den Mangel ins Zentrum, der - entgegen anderslautenden Erklärungen - als ein stabilisierendes Zentrum funktioniert. Da Lacan darüber hinaus auf der Unteilbarkeit des Briefes beharrt, folgt daraus für Derrida auch die Unteilbarkeit des Phallus:

Der Phallus, dank der Kastration, bleibt stets an seinem Platz, in der transzendentalen Topologie, von der wir sprachen weiter oben. Er ist hier unteilbar, und also unzerstörbar, wie der Brief, der davon die Statt hält. Und deshalb war die interessierte Voraussetzung, niemals demonstriert, der Materialität des Briefes als Unteilbarkeit unverzichtbar für diese beschränkte Ökonomie, für diese Zirkulation des Eigenen. Die Differenz, die mich hier interessiert, ist, daß [...] der Fehl nicht seinen Platz hat in der Dissemination. (Derrida 1987 [1975], 217)

Kurioserweise benutzt Lacan selbst nicht das Wort ,Phallus' in seiner Analyse, aber Derrida folgert, dass er letztendlich den Phallus im Sinn hat. Daher erhebt er gegen Lacan schließlich den massiven Vorwurf des Phallogozentrismus. Derrida geht davon aus, dass der Logos in der gesamten metaphysischen Tradition als heimlicher Mitwisser der geschlechtlichen Differenz fungierte, die im Phallogozentrismus mündete. Die Psychoanalyse degradierte den Phallus dann sowohl zum bloßen Signifikanten als auch zu einem Signifikanten des Mangels und folgte damit diesem traditionellen Pfad, indem sie ihn zum ,transzendentalen Signifikanten' ausrief, wenn auch ohne Signifikat. Das Zentrum mag zwar leer sein, aber es ist ein Zentrum, das reguliert und regiert.

Viertens findet sich die Psychoanalyse deshalb auch überall selbst wieder, wohin sie ihren Blick richtet. Sie kann unentwegt die gleiche Geschichte erkennen - die Geschichte von Ödipus, vom Phallus und der Kastration. Daher muss Derridas erster Satz im Text „Die Psychoanalyse, unterstellt, findet sich“ (Derrida 1987 [1975], 185) auch wie folgt gelesen werden: Die Psychoanalyse sucht und findet stets nur sich selbst, sie rechnet mit dem Erwartbaren, sie beschneidet die Literatur und deren disseminierende Kraft für ihre eigenen Zwecke. Deshalb erreicht der Brief auch immer sein Ziel. In der Dekonstruktion hingegen kann der Brief abhandenkommen, und gerade diese inhärente Möglichkeit des Verfehlens macht seine disseminative Natur aus. Derridas Kritik steht daher im Zentrum der poststrukturalistischen Auseinandersetzung mit der Psychoanalyse, insbeson- 
dere der feministischen. Darüber hinaus steht aber auch die Möglichkeit einer von der Psychoanalyse inspirierten Literaturwissenschaft zur Diskussion: Ist die Psychoanalyse an sich zwangsläufig unzureichend? Greift sie aufgrund ihrer strukturellen Mängel im Hinblick auf literarische Texte grundsätzlich zu kurz? Kann sie Literatur oder Literarizität gerecht werden, ohne sich von ihren wesentlichen Grundsätzen zu verabschieden? Diese großen prinzipiellen Fragen stehen mit den ungewöhnlichen Lektüren von Poes ungewöhnlicher Erzählung auf dem Spiel.

Doch stoßen Derridas Widersprüche gegen Lacans Poe-Lektüre ihrerseits auf Widerspruch, am einflussreichsten wohl in Barbara Johnsons bahnbrechendem Aufsatz The Frame of Reference: Poe, Lacan, Derrida (1977), mit dem gleichzeitig die Beziehung der beiden durch die ,Frau' trianguliert wird. Johnson zweifelt nicht nur Derridas Behauptungen an, sondern weist ihm auch nach, dass er viele der ,Verbrechen` selbst begeht, für die er Lacan anklagt (vgl. Johnson 1977, 465). Im Hinblick auf die Rahmung zeigt sie, dass Derrida Lacans Analyse selbst eigentümlich rahmt, indem er eine Anzahl textueller Voraussetzungen dieser Lektüre einfach außer Acht lässt. Dabei lasse seine eigene Rahmung von Poes Geschichte einiges zu wünschen übrig:

But if recognition is a form of blindness, a form of violence to the otherness of the object, it would seem that, by eliminating Lacan's suggestion of a possible complication of the phallic scheme, and by lying in wait between the brackets of the fireplace to catch the psychoanalyst at his own game, Derrida, too, is ,recognizing' rather than reading. And what he recognizes is, as he himself states it, a certain classical conception of psychoanalysis [...]. It would seem that the theoretical frame of reference which governs recognition is a constitutive element in the blindness of any interpretative insight. And it is precisely that frame of reference which allows the analyst to frame the author of the text he is reading for practices whose locus is simultaneously beyond the letter of the text and behind the vision of its reader. (Johnson 1977, 492)

Dass Derridas Kritik an Lacan über ihr Ziel hinausschießt, legen sowohl die Verlegenheit psychoanalytischer Lektüren im Besonderen als auch die Schwierigkeiten und toten Winkel literarischer Interpretationen im Allgemeinen offen. Denn jede Interpretation spielt mit dem Risiko von arbiträrer Rahmung, von Vereinheitlichung, von Konzentration auf bestimmte Textstellen, nur um andere zu vernachlässigen. Dieser kontingente oder gar gewalttätige Teil der Interpretation ist jedoch kein bloßer Makel, sondern ein Risiko, das die Produktivität einer Interpretation überhaupt erst ermöglicht. Jeder Rahmen kann und muss immer wieder neu gesetzt werden. Johnson gibt zwar zu, dass Lacan nur wenige auffällige Punkte der Erzählung für seine Zwecke berücksichtigt, doch bringt er damit neue 
und überraschende Einsichten ans Licht, die man eben nicht auf das psychoanalytische Masternarrativ, das jeder schon im Voraus kennt, reduzieren kann.

Das Wesentliche der Kontroverse hängt freilich von Lacans letztem Satz ab, demzufolge der Brief immer an seinem Ziel ankommt. Für Derrida steht diese Szene für eine vereinheitlichte Nachricht, die immer und unfehlbar ankommt, wie verworren auch immer der Weg ist. Doch was ist die Nachricht, die ankommt? Kann sie ausformuliert werden? Was vermittelt sie? Löst sie alle Mehrdeutigkeiten auf? Johnson schlägt eine Reihe möglicher Lesarten vor, die gerade aufgrund ihrer Mehrstimmigkeit funktionieren:

[I]t can mean ,the only message I can read is the one I send, ', wherever the letter is, is its destination, ', when a letter is read, it reads the reader,' ,the repressed always returns, ', I exist only as a reader of the other, ", the letter has no destination, ' and ,we all die.' It is not any one of these readings, but all of them and others in their very incompatibility, which repeat the letter in its way of reading the act of reading. Far from giving us the Seminar's final truth, these last words enact the impossibility of any ultimate analytical metalanguage. (Johnson 1977, 503)

In diesem Sinne kann man argumentieren, dass es paradoxerweise die Ankunft des Briefes an seinem Ziel statt sein Abhandenkommen ist, die Derridas über Lacans Lektüre verhängtes Todesurteil abwendet. Gehen der Brief und sein Ziel seiner Ankunft einfach voraus? Zeugt die Logik dahinter von einem ultimativen Ziel oder von einem ultimativen Zufall? Die Antworten auf diese Fragen können immer nur aufgeschoben werden.

\section{Kritik der Kritik}

Die Kontroverse führt ins Zentrum des Verhältnisses von Literatur, Poststrukturalismus und Psychoanalyse. Dass nämlich eben der Mangel von Bedeutung als die ultimative Bedeutung fungiert und damit alle Mehrdeutigkeiten zu einer Bedeutung vereinen kann, verweist auf ein Problem, für das es keine einfache Lösung gibt. Man kann argumentieren, dass die Signifikanten, die Derrida nutzt - Schreiben, Spur, Dissemination, différance, Textlichkeit, Rest -, genau die gleiche Neigung haben, als ,transzendentale Signifikanten` zu funktionieren, und damit die gleichen totalisierenden Effekte produzieren in just dem Moment, in dem sie der Totalisierung eine Absage erteilen. Sie mögen die Texte sehr wohl mit Bedeutung anreichern, während sie auf inhärente Fragmentierung schwören - auf den Rest, der nicht vereinheitlicht werden kann, auf Teilungen des Ganzen etc. Die Schwierigkeit im Umgang mit den totalisierenden Effekten des 
Signifikanten ist ein Problem, das Lacan vornehmlich in seinen späten Schriften verfolgt hat, als er etwa versucht, die Logik des pas-tout zu entwickeln (genau zu der Zeit, als Derrida in der Mitte der 1970er Jahre seine Kritik schrieb), und damit den von Derrida aufgeführten Problemen eine neue Wendung gibt.

Das Problem des Phallogozentrismus ist der faszinierendste und weitreichendste Teil von Derridas Behauptungen, wenn auch der abwegigste, da Lacan, wie bereits erwähnt, das Wort ,Phallus' überhaupt nicht verwendet und das Problem geschlechtlicher Differenz nicht anschneidet. Derrida behauptet indes, dass der Phallogozentrismus den allgemeinen Rahmen der Lacan'schen Analyse bildet. Damit verbinden sich nicht zuletzt ganz generelle Fragen: Ist die Psychoanalyse zuletzt die Auf- oder Abwertung des Phallus (und der Kastration etc.)? Ist sie seine Aufwertung hinter dem Schleier der Abwertung? Erhält sie am Ende nicht einfach die traditionelle phallogozentrische Weltanschauung, die sie also nur scheinbar kritisiert? Spielt es dem Phallogozentrismus nicht schon in die Hände, wenn man am Konzept des Phallus überhaupt festhält? Ist der Phallus die zentrale Figur, auch wenn man ihn als Signifikanten des Mangels begreift?

Die Antwort auf diese Fragen spaltet die Poststrukturalistinnen und Poststrukturalisten in zwei einander feindselig gegenüberstehende Lager, zwischen denen es keine Vermittlung, keine Versöhnung, keine Kompromisse, keine Neutralität oder Synthesen gibt. Es stehen die psychoanalytisch inspirierten (maßgeblich von Lacan beeinflussten) Theoretikerinnen und Theoretiker den Dekonstruktivisten sowie Anhängerinnen und Anhängern von Deleuze oder Foucault gegenüber. Diese Lagerbildung, die sich auf jeden Umgang mit Literatur in einem poststrukturalistischen Setting auswirkt, hat vor allem zur Abspaltung des Feminismus geführt, die Teil der poststrukturalistischen Wissenschaftsgeschichte ist. Während große und einflussreiche Theoretikerinnen wir Irigaray oder Cixous der Psychoanalyse mehr als kritisch gegenüberstehen, finden sich unter ihren Anhängerinnen ebenso herausragende Denkerinnen wie Shoshana Felman oder Joan Copjec, welche die Psychoanalyse eisern verteidigen; Kristeva und Judith Butler lassen sich in keinem Lager verorten.

Wir sind der Ansicht, dass es in der Tat eine inhärente Verbindung zwischen dem Konzept des Signifikanten und dem Konzept des Phallus bei Lacan gibt, einen ,phallischen Signifikanten'. In Lacans Essay La signification du phallus (1958; Die Bedeutung des Phallus), auf den sich Derrida oft bezieht, wird der Phallus als Schlüssel der Bedeutung entworfen (vgl. Lacan 2006 [1966]). Aber - und das ist ein großes ,Aber - der geschlechtliche Unterschied, wie Lacan ihn auffasst, kann nicht auf den Prozess der Bezeichnung reduziert und nicht von Signifikanten repräsentiert werden. Wenn Lacan darauf besteht, dass es ein Reales in der geschlechtlichen Differenz gibt, dann betrifft es das, was im Hinblick auf diesen Prozess irreduzibel ist, auf seine differenziellen und binären Oppositionen, 
auf sein Spiel von Präsenz und Absenz. Es geht also genau um das, was ,nicht aufhört, nicht geschrieben zu werden', um Lacans eigene Formel aus Encore zu bemühen (vgl. Lacan 1986 [1975]). Es kann nicht geschrieben werden, weil es vom Signifikanten beschlagnahmt wird und doch auf seinem Nicht-Geschrieben-Sein beharrt. Wenn es ein Problem geschlechtlicher Differenz in der Psychoanalyse gibt, dann dasjenige, dass sie nicht auf die Verbindung von Signifikanten und Phallus reduziert werden kann ( $\rightarrow$ III.10. DER FÜRST).

Die Psychoanalyse findet immer nur sich selbst, sie findet immer nur die gleichen alten Geschichten von Ödipus, Kastration, Phallus, Neurose, die immer neue Gestalten annehmen und denen - wie unterschiedlich auch immer sie sein mögen - die gleichen Grundsätze zugrunde liegen. Diese Gefahr lauert selbst an den Rändern der besten Interpretationen. Zwei Anmerkungen dazu: Wenn es erstens - das ist, was eine psychoanalytische Lektüre leistet, ohne etwas Unerwartetes zu produzieren, ohne neue Einsichten zu liefern, dann verdient sie jede Kritik, die ihr entgegenkommt. Der Test, dem sich jede gute Interpretation stellen muss, besteht jedoch nicht bloß darin, ob sie Neues produziert, sondern auch darin, ob sie die Theorie, die sie ,anwendet', durch ihre ,Anwendung' transformiert. Zweitens handelt es sich bei einer ,selbsterfüllenden Prophezeiung' nicht bloß um das Problem der Psychoanalyse, sondern um eine Gefahr aller philosophischen Literaturinterpretationen, die immer schon die bereits bestehende Theorie bestätigen und lediglich ihr eigenes Vorverständnis zutage fördern. Der Streit um Poes Geschichte ist bezeichnend und symptomatisch: Im Poststrukturalismus setzte die Psychoanalyse ihre kontroverse Geschichte fort. Die Literatur ist das Schlachtfeld, auf dem sich verschiedene Konzepte, Theorien, Behauptungen und Methoden erprobten, und es mag gut und gern so erscheinen, als seien die literarischen Texte selbst diesem Kampf zum Opfer gefallen, würden sie sich in ihrer Realität nicht jeder Form imaginärer oder symbolischer Aneignung widersetzen und immer neue Lektüren ermöglichen.

\section{Literatur}

Badiou, Alain. Das Abenteuer der französischen Philosophie. Seit den 60ern. Hrsg. von Peter Engelmann. Übers. von Paul Maercker. Wien 2015 [2012].

Barthes, Roland. Die Lust am Text. Übers. von Traugott König. Frankfurt a. M. 1974 [1973]. Barthes, Roland. Mythen des Alltags. Übers. von Horst Brühmann. Frankfurt a. M. 2010 [1957]. Barthes, Roland. S/Z. Übers. von Jürgen Hoch. 6. Aufl., Frankfurt a. M. 2012 [1970].

Bataille, Georges. Das Blau des Himmels. Übers. von Siegrid von Massenbach und Hans Naumann. München 1990 [1957].

Bonaparte, Marie. The Life and Works of Edgar Allan Poe. A Psychoanalytic Interpretation. New York 1971 [1949]. 
Brune, Carlo. Roland Barthes. Literatursemiologie und literarisches Schreiben. Würzburg 2003. Cusset, François. French Theory. Foucault, Derrida, Deleuze \& Cie et les mutations de la vie intellectuelle aux États-Unis. Paris 2003.

Deleuze, Gilles. „Sacher-Masoch und der Masochismus“ [1961]. Übers. von Gertrud Müller. Sacher-Masoch, Leopold von. Venus im Pelz. Mit einer Studie von Gilles Deleuze über den Masochismus. Frankfurt a. M. 1968: 167-295.

Deleuze, Gilles. Logik des Sinns. Aesthetica. Übers. von Bernhard Dieckmann. 2. Aufl., Frankfurt a. M. 1994 [1969].

Deleuze, Gilles. Differenz und Wiederholung. Übers. von Joseph Vogl. 2. Aufl., München 1997 [1972].

Deleuze, Gilles. Kritik und Klinik. Übers. von Joseph Vogl. Frankfurt a. M. 2000 [1993].

Deleuze, Gilles und Félix Guattari. Anti-Ödipus. Kapitalismus und Schizophrenie I. Übers. von Bernd Schwibs. 7. Aufl., Frankfurt a. M. 1995 [1972].

Deleuze, Gilles und Félix Guattari. Kafka. Für eine kleine Literatur. Übers. von Burkhart Kroeber. 9. Aufl., Frankfurt a. M. 2014 [1975].

Derrida, Jacques. „Freud und der Schauplatz der Schrift“ [1967]. Derrida, Jacques. Die Schrift und die Differenz. Übers. von Rodolphe Gasché. Frankfurt a. M. 1972: 302-350.

Derrida, Jacques. „Der Facteur der Wahrheit“ [1975]. Derrida, Jacques. Die Postkarte von Sokrates bis an Freud und jenseits. Übers. von Hans-Joachim Metzger. Berlin 1987: 183-281.

Derrida, Jacques. Grammatologie. Übers. von Hans Jörg Rheinberger und Hanns Zischler. Frankfurt a. M. 1983 [1967].

During, Simon. Foucault and Literature. Towards a Genealogy of Writing. New York 2012.

Felman, Shoshana (Hrsg.). Literature and Psychoanalysis. The Question of Reading: Otherwise. Baltimore 1982.

Felman, Shoshana. „On Reading Poetry. Reflections on the Limits and Possibilities of Psychoanalytic Approaches“. The Purloined Poe. Lacan, Derrida and Psychoanalytic Reading. Hrsg. von John P. Muller und William J. Richardson. Baltimore 1988: 133-155.

Foucault, Michel. Die Ordnung der Dinge. Eine Archäologie der Humanwissenschaften. Übers. von Ulrich Köppen. 13. Aufl., Frankfurt a. M. 1995 [1966].

Foucault, Michel. Sexualität und Wahrheit, Bd. 1: Der Wille zum Wissen. Übers. von Ulrich Raulff und Walter Seitter. 8. Aufl., Frankfurt a. M. 1995 [1976].

Geisenhanslüke, Achim. Textkulturen. Literaturtheorie nach dem Ende der Theorie. Paderborn 2015.

Jakobson, Roman. „Linguistik und Poetik“ [1960]. Jakobson, Roman. Poetik. Ausgewählte Aufsätze 1921-1971. Hrsg. und übers. von Elmar Holenstein und Tarcisius Schelbert. Frankfurt a. M. 1979: 83-121.

Johnson, Barbara. „The Frame of Reference. Poe, Lacan, Derrida“. Yale French Studies 55/56 (1977): 457-505.

Kafka, Franz. Tagebücher 1910-1923. Hrsg. von Max Brod. Frankfurt a. M. 1973.

Lacan, Jacques. „Das Seminar über E. A. Poes Der entwendete Brief“ [1966]. Lacan, Jacques. Schriften I. Hrsg. von Norbert Haas. Übers. von Rodolphe Gasché. Olten, Freiburg i. Br. 1973: 7-41.

Lacan, Jacques. Schriften, 3 Bde. Hrsg. von Norbert Haas. Übers. von Rodolphe Gasché. Olten, Freiburg i. Br. 1973-1980 [1966]. 
Lacan, Jacques. Das Seminar, Bd. XX: Encore (1972-1973). Hrsg. von Jacques-Alain Miller. Übers. von Norbert Haas, Vreni Haas und Hans-Joachim Metzger. 2. Aufl., Weinheim 1986 [1975].

Lacan, Jacques. Autres écrits. Paris 2001.

Lacan, Jacques. Le Séminaire, Bd. 23: Le Sinthome (1975-1976). Hrsg. von Jacques-Alain Miller. Paris 2005.

Lacan, Jacques. Das Seminar, Bd. V: Die Bildungen des Unbewussten (1957-1958). Hrsg. von Jacques-Alain Miller. Übers. von Hans-Dieter Gondek. Wien 2006 [1998].

Lacan, Jacques. Das Seminar, Bd. VIII: Die Übertragung (1960-1961). Hrsg. von Peter Engelmann. Übers. von Hans-Dieter Gondek. Wien 2008 [1991].

Lacan, Jacques. Le séminaire, Bd. VI: Le désir et son interprétation (1958-1959). Hrsg. von Jacques-Alain Miller. Paris 2013.

Lacan, Jacques. Das Seminar, Bd. VII: Die Ethik der Psychoanalyse (1959-1960). Hrsg. von Norbert Haas und Hans-Joachim Metzger. Übers. von Norbert Haas. Wien, Berlin 2016 [1986].

Lecercle, Jean-Jacques. Badiou and Deleuze Read Literature. Edinburgh 2012.

Lindorfer, Bettine. Roland Barthes. Zeichen und Psychoanalyse. München 1998.

Macksey, Richard (Hrsg.). The Structuralist Controversy. Baltimore 1972.

Neumann, Gerhard. Kulturwissenschaftliche Hermeneutik. Interpretieren nach dem Poststrukturalismus. Freiburg i. Br. 2014.

Poe, Edgar Allan. Selected Writings. Hrsg. von David Galloway. New York 1967.

Sawyer, Dylan. Lyotard, Literature and the Trauma of the Differend. London 2014.

Schuster, Aaron. The Trouble with Pleasure. Deleuze and Psychoanalysis. Cambridge/Mass. 2016.

Serres, Michel. L'hermaphrodite. Sarrasine sculpteur. Paris 1987.

Zanetti, Sandro. „Literaturwissenschaftliches Schreiben zwischen Mimesis und Abstraktion. Von Jean Leclerc zu Peter Szondi und Roland Barthes“. Internationales Archiv für Sozialgeschichte der deutschen Literatur 40.2 (2015): 348-373.

Zupančič, Alenka. The Ethics of the Real. London 2000. 